\title{
Faculty Performance Improvement through Effective Human Resource Management Practices
}

\author{
Thanikachalam Vedhathiri \\ (vthani2025@gmail.com) \\ Former Professor and HOD, \\ Center for International Affairs, \\ National Institute of Technical teachers training \\ and Research, Chennai-600113, India
}

\begin{abstract}
Most of the universities do not have separate human resource management departments like corporates. Many higher educational institutes follow the discretions of the Chief Executive Officer (CEO). The faculties' outstanding contributions are not reviewed and rewarded. They do not provide needed initial funds for undertaking consultancy projects and supporting staff. Most of them shift the project leaders to underqualified faculty who are their coteries. Due to this the key performing faculty teams lose the motivation and struggle to contribute to the growth of knowledge- based human capital. Hence, it is planned to review the best human resource management practices of award -winning companies identified and added in Fortune 500 list, the best performing universities and identify the best practices in facilitating the faculty members and managing life- balance. This will help the universities to remodel their faculty management practices and accelerate their contribution to human and knowledge capital.
\end{abstract}

Keywords: Best human resource management practices of outstanding corporates and universities, Desired improvements in human resource management, Lessons to be learned and Initiatives in life-balance practices, Outcomes based on the HRM in the colleges.

\section{Introduction}

In almost all educational institutes there is NO department for human resource management. The existing administrative office handles all issues regarding the "people (faculty) management" without any deep concern for the growth of human resources (scientists, researchers, leaders or innovators). A few high performing universities started getting the feedback from their faculty members to correct the wrong policies.

In many developing countries, most of the CEOs of higher education do not nominate the faculty for undergoing training or internship offered by various organizations like United Nations Education and Scientific Council (UNESCO), United Nations Development Program (UNDP), World Bank and ongoing projects of foreign universities under bilateral agreements. They even do not permit the outstanding

faculty to present papers in the international conferences even if the faculty gets invitation and funding. They tactically send the nominations after the completion of the last date for receiving the applications. Some will forward the application but later they deny relieving the faculty to join the programs. There is no proper human resource management. Also, many CEOs do not even consider the need for life balance. Due this the institute loses opportunities to get the faculty updated and serve the institute based on their improved competencies. Many CEOs do not reward the faculty for their outstanding accomplishments.

Such practices not only full down the key performing faculty members but also substantially lowers the competitiveness of the region. In the knowledge based global economy, the performance of the engineering colleges is to be improved through appropriate human management strategies. This research work centers around the investigation, identification, validation and suggestion of best methods to improve the outcomes of the graduates through faculties.

\section{Objectives}

To review the best practices of award- winning companies and best performing universities/ institutions in implementing outstanding human resource management initiates and the impact of the business/ academic success.

To identify needed human resource practices to accelerate the performance and improve the knowledge capital and human capitals.

To suggest the best practices to incorporate human resource development initiatives similar to the best performing universities/ institutions.

Review of Human Resource Practices of AwardWinning Companies 
The outstanding companies have allowed their employees to undergo training programs. The companies also conduct required training to continuously improve the skills and competencies. The following study has been undertaken to identify the practices of 30 companies which were recognized for human resources efforts and practices. The companies brought many innovations in human resource development. They emphasized on quality and diversity within the workplace. They further provided employee incentives and, onsite perks and rewards for high performance. Their recruitment efforts, regular evaluation, transparency and employee engagement will provide guidelines for educational administrators. 30 well recognized companies are considered for this study. Most of them were earned Glassdoor Employee's Choice Award as One of the Best Places to Work, Fortune's 500 Best Companies to work for, Sunday Times recognition etc. The findings are presented in Table-1. Based on this, a set of lessons to be learned are derived. If these lessons are practiced by the

Table-1 Implementation of best practices work-life balances

\begin{tabular}{|c|c|c|}
\hline University & $\begin{array}{c}\text { Vision and } \\
\text { Faculty Needs }\end{array}$ & $\begin{array}{c}\text { Implementati } \\
\text { on }\end{array}$ \\
\hline $\begin{array}{l}\text { University of } \\
\text { Pittsburgh, } \\
\text { USA }\end{array}$ & $\begin{array}{l}\text { The university } \\
\text { is committed to } \\
\text { helping their } \\
\text { most important } \\
\text { resources- their } \\
\text { faculty and } \\
\text { staff. The } \\
\text { faculty } \\
\text { influences } \\
\text { policies and } \\
\text { programs } \\
\text { through } \\
\text { participation. }\end{array}$ & $\begin{array}{l}\text { To help } \\
\text { balance work } \\
\text { achievement } \\
\text { and life } \\
\text { activities, } \\
\text { university } \\
\text { offers a variety } \\
\text { of programs, } \\
\text { services to help } \\
\text { employees } \\
\text { attain a healthy } \\
\text { balanced } \\
\text { lifestyle. }\end{array}$ \\
\hline $\begin{array}{l}\text { Iowa State } \\
\text { University } \\
\text { (ISU), USA }\end{array}$ & $\begin{array}{l}\text { The ISU } \\
\text { Advance } \\
\text { program } \\
\text { recognizes the } \\
\text { importance of } \\
\text { work-life issues } \\
\text { to recruitment } \\
\text { and retention of } \\
\text { excellent } \\
\text { faculty. }\end{array}$ & $\begin{array}{l}\text { In partnership } \\
\text { with the Office } \\
\text { of the Senor } \\
\text { Vice President, } \\
\text { the ISU } \\
\text { Advance } \\
\text { program has } \\
\text { developed } \\
\text { resources and } \\
\text { initiatives to } \\
\text { improve the } \\
\text { work-life at } \\
\text { ISU. }\end{array}$ \\
\hline $\begin{array}{l}\text { Boise State } \\
\text { University, } \\
\text { USA }\end{array}$ & $\begin{array}{l}\text { This university } \\
\text { is committed to } \\
\text { helping its } \\
\text { faculty } \\
\text { members } \\
\text { balance the } \\
\text { responsibilities } \\
\text { of their }\end{array}$ & $\begin{array}{l}\text { The university } \\
\text { resources will } \\
\text { provide them } \\
\text { with } \\
\text { information } \\
\text { they need to } \\
\text { ensure a } \\
\text { healthy }\end{array}$ \\
\hline
\end{tabular}

higher education institutes, the faculty members can contribute to the growth of knowledge capital.

\section{Vision and Initiatives of Selected Outstanding Universities}

In today's fast-paced world, maintaining a healthy balance between a successful career and a fulfilling personal life can be a challenge. Increasing demands on the faculty time- both in the workplace and at homecan create significant stress. The faculty members are pulled in a dozen directions. Classes, committees, research work, sponsored projects, research guidance, bid document preparation, and more can threaten to drown even the most resilient of the faculty. They need more guidance, assistance, and resources to manage their problems. The high performing universities have taken efforts to help their faculty and staff to manage their work life balance. Some of the outstanding decisions and their implementation are presented in the following section.

\begin{tabular}{|c|c|c|}
\hline & $\begin{array}{l}\text { professional } \\
\text { and balance } \\
\text { lives. }\end{array}$ & $\begin{array}{l}\text { work/life } \\
\text { balance. Adult } \\
\text { and Elder care. } \\
\text { Center for } \\
\text { Study of } \\
\text { Aging; } \\
\text { Powerful Tools } \\
\text { for Caregivers. }\end{array}$ \\
\hline $\begin{array}{l}\text { University of } \\
\text { Pennsylvania, } \\
\text { USA }\end{array}$ & $\begin{array}{l}\text { Work-Life } \\
\text { Resources Penn } \\
\text { is committed to } \\
\text { supporting their } \\
\text { faculty and } \\
\text { staff as they } \\
\text { balance the } \\
\text { competing } \\
\text { demands of } \\
\text { work and } \\
\text { personal life. }\end{array}$ & $\begin{array}{l}\text { The university } \\
\text { offers a wide } \\
\text { variety of } \\
\text { programs and } \\
\text { resources to } \\
\text { help them care } \\
\text { for health, their } \\
\text { family and } \\
\text { their work-life } \\
\text { balance. }\end{array}$ \\
\hline $\begin{array}{l}\text { University } \\
\text { College, } \\
\text { Dublin, Ireland }\end{array}$ & $\begin{array}{l}\text { The university } \\
\text { aims to } \\
\text { promote work } \\
\text { life balance for } \\
\text { all staff } \\
\text { members. }\end{array}$ & $\begin{array}{l}\text { The university } \\
\text { has range of } \\
\text { flexible } \\
\text { working and } \\
\text { leaf options } \\
\text { available for to } \\
\text { staff. }\end{array}$ \\
\hline $\begin{array}{l}\text { University of } \\
\text { Pittsburgh, } \\
\text { USA }\end{array}$ & $\begin{array}{l}\text { Life Solutions: } \\
\text { University } \\
\text { Family and } \\
\text { Staff } \\
\text { Assistance } \\
\text { Program. }\end{array}$ & $\begin{array}{l}\text { It provides a } \\
\text { broad range of } \\
\text { services to } \\
\text { assist the } \\
\text { university } \\
\text { employees and } \\
\text { their household } \\
\text { members to } \\
\text { balance work } \\
\text { and the stresses }\end{array}$ \\
\hline
\end{tabular}




\begin{tabular}{|c|c|c|}
\hline & & $\begin{array}{l}\text { of daily life. } \\
\text { Life solutions } \\
\text { offer } \\
\text { personalized } \\
\text { care services, } \\
\text { 24-hour } \\
\text { support. }\end{array}$ \\
\hline $\begin{array}{l}\text { The Rutgers } \\
\text { University, } \\
\text { USA }\end{array}$ & $\begin{array}{l}\text { The } \\
\text { university's } \\
\text { Faculty and } \\
\text { Staff and Staff } \\
\text { Assistance } \\
\text { Program } \\
\text { (FSAP) } \\
\text { provides } \\
\text { comprehensive } \\
\text { counseling and } \\
\text { referral service } \\
\text { to the } \\
\text { university } \\
\text { community. }\end{array}$ & $\begin{array}{l}\text { The Crime } \\
\text { Victim } \\
\text { Assistance } \\
\text { Program offers } \\
\text { a range of } \\
\text { services to } \\
\text { assist the } \\
\text { persons with } \\
\text { aftermath of } \\
\text { victimization. } \\
\text { Work/life } \\
\text { Balance } \\
\text { Resources are } \\
\text { available for } \\
\text { employees and } \\
\text { retirees. }\end{array}$ \\
\hline $\begin{array}{l}\text { Bridgewater } \\
\text { State } \\
\text { University, } \\
\text { USA }\end{array}$ & $\begin{array}{l}\text { The university } \\
\text { established the } \\
\text { Faculty } \\
\text { Advocacy } \\
\text { Network } \\
\text { (FAN). }\end{array}$ & $\begin{array}{l}\text { FAN provides } \\
\text { one to one } \\
\text { collaborative } \\
\text { and group } \\
\text { support to } \\
\text { Bridgewater } \\
\text { University } \\
\text { faculty at all } \\
\text { phases of their } \\
\text { careers. } \\
\text { Through } \\
\text { networking } \\
\text { partnerships, } \\
\text { FAN offers } \\
\text { guidance and } \\
\text { practical } \\
\text { assistance with } \\
\text { short-term and } \\
\text { long-term goal } \\
\text { setting and } \\
\text { balancing. }\end{array}$ \\
\hline $\begin{array}{l}\text { College of } \\
\text { Engineering, } \\
\text { Guindy, Anna } \\
\text { University, } \\
\text { Chennai, India }\end{array}$ & $\begin{array}{l}\text { Implements } \\
\text { various faculty } \\
\text { development } \\
\text { programs. } \\
\text { Nominates the } \\
\text { faculty for } \\
\text { international } \\
\text { programs and } \\
\text { provides } \\
\text { funding. }\end{array}$ & $\begin{array}{l}\text { Recognizes the } \\
\text { outstanding } \\
\text { achievement in } \\
\text { internal } \\
\text { revenue } \\
\text { generation and } \\
\text { rewards the } \\
\text { faculty. } \\
\text { Supports the } \\
\text { consultancy } \\
\text { works, and } \\
\text { research } \\
\text { projects. }\end{array}$ \\
\hline $\begin{array}{l}\text { Indian Institute } \\
\text { of Technology- }\end{array}$ & $\begin{array}{l}\text { The Institute } \\
\text { wanted to reach } \\
\text { excellence in }\end{array}$ & $\begin{array}{l}\text { The Institute } \\
\text { selected the } \\
\text { best students }\end{array}$ \\
\hline
\end{tabular}

\begin{tabular}{|c|c|c|}
\hline $\begin{array}{l}\text { Madras, } \\
\text { Chennai, India }\end{array}$ & $\begin{array}{l}\text { teaching, } \\
\text { research, } \\
\text { entrepreneurshi } \\
\text { p, student } \\
\text { placement, and } \\
\text { consultancy. } \\
\text { The Institute } \\
\text { reached the } \\
\text { highest level in } \\
\text { academic } \\
\text { performance in } \\
\text { the country by } \\
\text { following best } \\
\text { practices in } \\
\text { faculty } \\
\text { management. }\end{array}$ & $\begin{array}{l}\text { through } \\
\text { national } \\
\text { entrance } \\
\text { examination } \\
\text { selected the } \\
\text { best faculty, } \\
\text { constructed } \\
\text { excellent } \\
\text { quarters, } \\
\text { schools, } \\
\text { shopping } \\
\text { center, bus } \\
\text { transport and } \\
\text { water supply. } \\
\text { This Institute } \\
\text { awarded } \\
\text { pension to all } \\
\text { the faculty and } \\
\text { staff who opt } \\
\text { ed for the same } \\
\text { very late. }\end{array}$ \\
\hline $\begin{array}{l}\text { Agriculture } \\
\text { College and } \\
\text { Research } \\
\text { Institute, } \\
\text { Madurai, Tamil } \\
\text { Nadu, India }\end{array}$ & $\begin{array}{l}\text { The university } \\
\text { wanted to } \\
\text { undertake } \\
\text { outstanding } \\
\text { research work } \\
\text { in crop } \\
\text { production and } \\
\text { extend the } \\
\text { technology in } \\
\text { that climate. } \\
\text { The faculty will } \\
\text { be performing } \\
\text { all class work, } \\
\text { research and } \\
\text { extension work. }\end{array}$ & $\begin{array}{l}\text { The university } \\
\text { provided } \\
\text { excellent } \\
\text { quarters, water } \\
\text { supply, } \\
\text { electricity, bus } \\
\text { transport for } \\
\text { the faculty and } \\
\text { the staff, an } \\
\text { extension } \\
\text { center of a } \\
\text { bank, an } \\
\text { outpatient ward } \\
\text { and a doctor. } \\
\text { The College is } \\
\text { selling paddy, } \\
\text { vegetables, } \\
\text { fruits and } \\
\text { chicken to the } \\
\text { residents at } \\
\text { cost price. }\end{array}$ \\
\hline $\begin{array}{l}\text { Bhaktavatchala } \\
\text { m Polytechnic } \\
\text { College, } \\
\text { Kanchipuram, } \\
\text { Tamil Nadu, } \\
\text { India }\end{array}$ & $\begin{array}{l}\text { The } \\
\text { management } \\
\text { wanted to } \\
\text { improve the } \\
\text { skills of the } \\
\text { students and } \\
100 \% \\
\text { employment } \\
\text { through a team } \\
\text { of dedicated } \\
\text { faculty } \\
\text { members who } \\
\text { have been } \\
\text { trained. They } \\
\text { also ward the } \\
\text { best performed } \\
\text { student of the } \\
\text { state. }\end{array}$ & $\begin{array}{l}\text { The } \\
\text { management } \\
\text { recognized the } \\
\text { outstanding } \\
\text { achievement of } \\
\text { the } \\
\text { departments as } \\
\text { follows: Prizes } \\
\text { were } \\
\text { distributed to } \\
\text { the faculty } \\
\text { when they } \\
\text { produce } 100 \% \\
\text { pass in the } \\
\text { Board } \\
\text { examination. If } \\
\text { all the students } \\
\text { obtain the job }\end{array}$ \\
\hline
\end{tabular}




\begin{tabular}{|l|l|}
\hline & within three \\
& months, the \\
faculty of the \\
department \\
was awarded \\
silver medal. If \\
\\
& a student \\
received the \\
highest state \\
rank, the \\
department \\
will be \\
awarded a \\
\end{tabular}

Table-2 Innovations implemented by 30 companies and the lessons to learn

\begin{tabular}{|c|c|c|c|}
\hline & $\begin{array}{l}\text { Company, Web } \\
\text { site, Employees. }\end{array}$ & $\begin{array}{l}\text { Innovati } \\
\text { ons } \\
\text { Impleme } \\
\text { nted }\end{array}$ & $\begin{array}{l}\text { Lessons to } \\
\text { learn }\end{array}$ \\
\hline $\begin{array}{l}3 \\
0\end{array}$ & $\begin{array}{l}\text { FedEx Corporation, } \\
\text { Multinational } \\
\text { Courier Services, } \\
\text { Tennessee, } \\
\text { USA } \\
\text { https://www.about.v } \\
\text { an.fedex.com/ } \\
\text { our-story/company- } \\
\text { structure/ } \\
\text { corporate-fact-sheet/ } \\
\text { Employees: } 42500\end{array}$ & $\begin{array}{l}\text { Vision: } \\
\text { "People- } \\
\text { Service- } \\
\text { Profit" } \\
\text { Excellent } \\
\text { care of } \\
\text { employee } \\
\text { s breeds } \\
\text { excellent } \\
\text { service. } \\
\text { To track } \\
\text { employee } \\
\text { relations, } \\
\text { FedEx } \\
\text { offers an } \\
\text { annual } \\
\text { survey } \\
\text { and } \\
\text { feedback } \\
\text { action } \\
\text { program. } \\
\text { Employe } \\
\text { es } \\
\text { provide } \\
\text { valuable } \\
\text { feedback } \\
\text { and } \\
\text { managem } \\
\text { ent meets } \\
\text { to discuss } \\
\text { results. } \\
\text { The } \\
\text { program } \\
\text { allows } \\
\text { problem } \\
\text { assessme } \\
\text { nt and } \\
\text { resolution }\end{array}$ & $\begin{array}{l}\text { "Faculty- } \\
\text { Service- } \\
\text { Human \& } \\
\text { Knowledg } \\
\text { e } \\
\text { Capitals". } \\
\text { Conduct } \\
\text { an annual } \\
\text { survey and } \\
\text { feedback } \\
\text { action } \\
\text { program. } \\
\text { Resolve } \\
\text { the } \\
\text { problems } \\
\text { amicably. } \\
\text { Encourage } \\
\text { advanced } \\
\text { and } \\
\text { Interdiscip } \\
\text { linary } \\
\text { Research. } \\
\text { Programs. }\end{array}$ \\
\hline
\end{tabular}

\begin{tabular}{|c|c|c|c|}
\hline & & $\begin{array}{l}\text { opportuni } \\
\text { ties. } \\
\text { FedEx } \\
\text { Corporati } \\
\text { on } \\
\text { provides } \\
\text { an } \\
\text { efficient } \\
\text { way to } \\
\text { address } \\
\text { problems } \\
\text { in order } \\
\text { to keep } \\
\text { the } \\
\text { communi } \\
\text { ty } \\
\text { balanced. }\end{array}$ & \\
\hline $\begin{array}{l}2 \\
9\end{array}$ & $\begin{array}{l}\text { AMEX } \\
\text { American Express, } \\
\text { USA } \\
\text { www.american.expre } \\
\text { ss.com } \\
\text { Employees: } 59000\end{array}$ & $\begin{array}{l}\text { Focuses } \\
\text { service } \\
\text { on world- } \\
\text { class } \\
\text { customer. } \\
\text { Training } \\
\text { is critical. } \\
\text { Conscient } \\
\text { ious } \\
\text { hiring } \\
\text { practices } \\
\text { and } \\
\text { layoff } \\
\text { procedure } \\
\text { s. } \\
\text { Communi } \\
\text { cation is } \\
\text { taught } \\
\text { among } \\
\text { staff and } \\
\text { accompli } \\
\text { shed by } \\
\text { the } \\
\text { implemen } \\
\text { tation of } \\
\text { an open- } \\
\text { space } \\
\text { work area } \\
\text { for } \\
\text { employee } \\
\text { s. } \\
\text { Managem } \\
\text { ent and } \\
\text { leadershi } \\
\text { p teams } \\
\text { practice } \\
\text { open- } \\
\text { dooor } \\
\text { policies } \\
\text { and } \\
\text { welcome } \\
\text { the voice } \\
\text { of even }\end{array}$ & $\begin{array}{l}\text { Implement } \\
\text { open space } \\
\text { work area } \\
\text { for the } \\
\text { faculty } \\
\text { and } \\
\text { students. } \\
\text { Encourage } \\
\text { faculty to } \\
\text { work in } \\
\text { the labs } \\
\text { and } \\
\text { workshops } \\
\text { during } \\
\text { holidays } \\
\text { and after } \\
\text { office } \\
\text { hours. } \\
\text { Open the } \\
\text { library for } \\
24 x 7 \text {. } \\
\text { Implement } \\
\text { open door- } \\
\text { policy. Get } \\
\text { the new } \\
\text { proposals } \\
\text { for } \\
\text { developme } \\
\text { nt. }\end{array}$ \\
\hline
\end{tabular}




\begin{tabular}{|c|c|c|c|}
\hline & & $\begin{array}{l}\text { entry- } \\
\text { level } \\
\text { trainees. } \\
\text { As a } \\
\text { proactive } \\
\text { effort to } \\
\text { maintain } \\
\text { employee } \\
\text { relations } \\
\text { during } \\
\text { layoffs, } \\
\text { AMX } \\
\text { offers } \\
\text { transpare } \\
\text { nt } \\
\text { severance } \\
\text { packages } \\
\text { that are } \\
\text { both } \\
\text { generous } \\
\text { and } \\
\text { helpful in } \\
\text { relocating } \\
\text { those } \\
\text { affected } \\
\text { by the } \\
\text { change. }\end{array}$ & \\
\hline $\begin{array}{l}2 \\
8\end{array}$ & $\begin{array}{l}\text { Oxfam } \\
\text { (Oxford Committee } \\
\text { for Famine Relief), } \\
\text { International NGO, } \\
\text { UK } \\
\text { www.oxfam.org.uk }\end{array}$ & $\begin{array}{l}\text { Oxfam } \\
\text { dominate } \\
\text { s when it } \\
\text { comes to } \\
\text { managem } \\
\text { ent, } \\
\text { leadershi } \\
\text { p, } \\
\text { developm } \\
\text { ent and } \\
\text { training } \\
\text { people. } \\
\text { They see } \\
\text { people } \\
\text { developm } \\
\text { ent as a } \\
\text { critical } \\
\text { compone } \\
\text { nt to } \\
\text { staying } \\
\text { afloat. } \\
\text { They } \\
\text { value the } \\
\text { training } \\
\text { of people } \\
\text { as an } \\
\text { important } \\
\text { retention } \\
\text { tool } \\
\text { among a } \\
\text { sector } \\
\text { that } \\
\text { typically }\end{array}$ & $\begin{array}{l}\text { Faculty } \\
\text { developme } \\
\text { nt is } \\
\text { essential. } \\
\text { Establish } \\
\text { in-house } \\
\text { faculty } \\
\text { developme } \\
\text { nt } \\
\text { programs. } \\
\text { Encourage } \\
\text { participati } \\
\text { on in } \\
\text { MOOCs } \\
\text { (online } \\
\text { programs) } \\
\text { of } \\
\text { Coursera, } \\
\text { EdX, } \\
\text { WBI, } \\
\text { Swayam } \\
\text { of } \\
\text { NITTTRs. }\end{array}$ \\
\hline
\end{tabular}

\begin{tabular}{|c|c|c|c|}
\hline & & $\begin{array}{l}\text { struggles } \\
\text { to } \\
\text { compete } \\
\text { on } \\
\text { salaries } \\
\text { with } \\
\text { business. } \\
\text { They } \\
\text { train their } \\
\text { people to } \\
\text { regard } \\
\text { the same } \\
\text { beliefs, } \\
\text { and as a } \\
\text { result, are } \\
\text { making } \\
\text { an impact } \\
\text { and } \\
\text { differenc } \\
\text { e in the } \\
\text { HR } \\
\text { arena. }\end{array}$ & \\
\hline $\begin{array}{l}2 \\
7\end{array}$ & $\begin{array}{l}\text { Prudential } \\
\text { (Financial } \\
\text { Institution), USA } \\
\text { www.prudential.com } \\
\text { Employees: } 49705\end{array}$ & $\begin{array}{l}\text { They } \\
\text { attribute } \\
\text { their } \\
\text { human } \\
\text { resource } \\
\text { success } \\
\text { to their } \\
\text { transpare } \\
\text { ncy and } \\
\text { ability to } \\
\text { share } \\
\text { their } \\
\text { story. } \\
\text { The } \\
\text { company' } \\
\text { s history } \\
\text { is shared } \\
\text { with its } \\
\text { entire } \\
\text { staff, } \\
\text { discussin } \\
\text { g the } \\
\text { highs and } \\
\text { lows, } \\
\text { challenge } \\
\text { s and } \\
\text { successes } \\
\text {, with } \\
\text { every } \\
\text { person. } \\
\text { The } \\
\text { company } \\
\text { keeps an } \\
\text { open } \\
\text { mind in } \\
\text { HR } \\
\text { processes } \\
\text { and }\end{array}$ & $\begin{array}{l}\text { Be } \\
\text { transparent } \\
\text { and share } \\
\text { the } \\
\text { perspectiv } \\
\text { e plan and } \\
\text { involve } \\
\text { the faculty } \\
\text { in the } \\
\text { strategic } \\
\text { planning. } \\
\text { Circulate } \\
\text { the letters } \\
\text { on } \\
\text { preparing } \\
\text { projects. }\end{array}$ \\
\hline
\end{tabular}




\begin{tabular}{|c|c|c|c|}
\hline & & $\begin{array}{l}\text { welcomes } \\
\text { change } \\
\text { and } \\
\text { innovatio } \\
n \text { when } \\
\text { needed. } \\
\text { Their } \\
\text { open } \\
\text { mind is } \\
\text { keeping } \\
\text { them } \\
\text { ahead of } \\
\text { the rest. }\end{array}$ & \\
\hline $\begin{array}{l}2 \\
6\end{array}$ & $\begin{array}{l}\text { Eastman Chemical } \\
\text { Company } \\
\text { Tennessee, USA } \\
\text { www.eastman.com/c } \\
\text { ompany/ } \\
\text { About-Eastman/ } \\
\text { Employees: } 14500\end{array}$ & $\begin{array}{l}\text { Company } \\
\text { values } \\
\text { the input } \\
\text { and voice } \\
\text { of its } \\
\text { employee } \\
\text { s. } \\
\text { Believes } \\
\text { that when } \\
\text { their } \\
\text { people } \\
\text { love their } \\
\text { work and } \\
\text { enjoy } \\
\text { working } \\
\text { with one } \\
\text { another, } \\
\text { remarkab } \\
\text { le things } \\
\text { happen. } \\
\text { The } \\
\text { award } \\
\text { received } \\
\text { by it was } \\
\text { based on } \\
\text { their } \\
\text { employee } \\
\text { rate of } \\
\text { satisfacti } \\
\text { on with } \\
\text { the } \\
\text { company, } \\
\text { as well as } \\
\text { critical } \\
\text { workplac } \\
\text { e factors } \\
\text { like } \\
\text { compensa } \\
\text { tion, } \\
\text { benefits, } \\
\text { advance } \\
\text { ment } \\
\text { opportuni } \\
\text { ty, work- } \\
\text { life } \\
\text { balance, } \\
\text { career }\end{array}$ & $\begin{array}{l}\text { Encourage } \\
\text { s the } \\
\text { faculty to } \\
\text { plan } \\
\text { courses } \\
\text { and } \\
\text { programs. } \\
\text { Listen to } \\
\text { the views } \\
\text { of the } \\
\text { faculty } \\
\text { association } \\
\text { Promote } \\
\text { the faculty } \\
\text { based on } \\
\text { their world } \\
\text { class } \\
\text { contributio } \\
\text { ns. }\end{array}$ \\
\hline
\end{tabular}

\begin{tabular}{|c|c|c|c|}
\hline & & $\begin{array}{l}\text { culture, } \\
\text { and } \\
\text { access to } \\
\text { senior } \\
\text { managem } \\
\text { ent. }\end{array}$ & \\
\hline $\begin{array}{l}2 \\
5\end{array}$ & $\begin{array}{l}\text { Wegmans Food } \\
\text { Markets, Inc. } \\
\text { (American } \\
\text { Supermarket Chain), } \\
\text { NY, USA } \\
\text { www.wegmans.com } \\
\text { Employees: } 49000\end{array}$ & $\begin{array}{l}\text { The } \\
\text { emphasis } \\
\text { it puts on } \\
\text { taking } \\
\text { care of } \\
\text { both its } \\
\text { employee } \\
\text { s and } \\
\text { customer } \\
\text { s. }\end{array}$ & $\begin{array}{l}\text { Take care } \\
\text { of the } \\
\text { faculty } \\
\text { and } \\
\text { students } \\
\text { by } \\
\text { accident } \\
\text { insurance } \\
\text { when they } \\
\text { undertake } \\
\text { outstation } \\
\text { trips for } \\
\text { consultanc } \\
\text { y projects. }\end{array}$ \\
\hline $\begin{array}{l}2 \\
4\end{array}$ & $\begin{array}{l}\text { Alliance Boots } \\
\text { GmbH } \\
\text { (Pharmaceuticals } \\
\text { Company), } \\
\text { Switzerland } \\
\text { www.bloomberg.co } \\
\text { m/profile/ } \\
\text { company/360963iz:S } \\
\text { W } \\
\text { Employees: >120000 }\end{array}$ & $\begin{array}{l}\text { Excels in } \\
\text { staffing } \\
\text { supportiv } \\
\text { e } \\
\text { managers } \\
\text { improvin } \\
\text { g } \\
\text { colleague } \\
\text { relations, } \\
\text { promotin } \\
\text { g a stress- } \\
\text { free work } \\
\text { environm } \\
\text { ent, and } \\
\text { promotin } \\
\text { g } \\
\text { philanthr } \\
\text { opy } \\
\text { through } \\
\text { outreach } \\
\text { activities. } \\
\text { Offers } \\
\text { vocationa } \\
1 \text { training } \\
\text { for its } \\
\text { entry- } \\
\text { level } \\
\text { employee } \\
\text { s, } \\
\text { offering } \\
\text { room to } \\
\text { move } \\
\text { upward } \\
\text { over } \\
\text { time. } \\
\text { Offers } \\
\text { formal } \\
\text { accreditat } \\
\text { ion for }\end{array}$ & $\begin{array}{l}\text { Improve } \\
\text { interperso } \\
\text { nal } \\
\text { relations. } \\
\text { Encourage } \\
\text { faculty } \\
\text { get-to- } \\
\text { gather, } \\
\text { picnics, } \\
\text { retreats, } \\
\text { and field } \\
\text { trips. } \\
\text { Promote } \\
\text { stress free } \\
\text { work } \\
\text { environme } \\
\text { nt. } \\
\text { Contribute } \\
\text { to } \\
\text { philanthro } \\
\text { py. } \\
\text { Encourage } \\
\text { best } \\
\text { performers } \\
\text { by } \\
\text { promotion. }\end{array}$ \\
\hline
\end{tabular}




\begin{tabular}{|c|c|c|c|}
\hline & & $\begin{array}{l}\text { work } \\
\text { within } \\
\text { the } \\
\text { communi } \\
\text { ty. }\end{array}$ & \\
\hline $\begin{array}{l}2 \\
3\end{array}$ & $\begin{array}{l}\text { Bain and Company } \\
\text { (Business and Health } \\
\text { Products), } \\
\text { USA } \\
\text { www.bain.com } \\
\text { Employees: } 10500\end{array}$ & $\begin{array}{l}\text { Work } \\
\text { culture: } \\
\text { Hard- } \\
\text { working } \\
\text { people } \\
\text { who love } \\
\text { what they } \\
\text { do. Bain } \\
\text { is a } \\
\text { magnet } \\
\text { for top } \\
\text { talent and } \\
\text { employee } \\
\text { s who } \\
\text { stay the } \\
\text { course. } \\
\text { The team } \\
\text { culture } \\
\text { allows all } \\
\text { members } \\
\text { to fully } \\
\text { contribut } \\
\text { e ideas, } \\
\text { support } \\
\text { each } \\
\text { other, and } \\
\text { provide } \\
\text { inspiratio } \\
\text { n as a } \\
\text { communi } \\
\text { ty. } \\
\text { Company } \\
\text { focuses } \\
\text { on the } \\
\text { complete- } \\
\text { employee } \\
\text {; } \\
\text { combinin } \\
\text { g } \\
\text { training, } \\
\text { mentorin } \\
\text { g, and } \\
\text { flexibility } \\
\text { among } \\
\text { work } \\
\text { schedules }\end{array}$ & $\begin{array}{l}\text { Hard } \\
\text { working } \\
\text { faculty } \\
\text { love what } \\
\text { they do. } \\
\text { Encourage } \\
\text { bidding } \\
\text { for global } \\
\text { consultanc } \\
\text { y projects } \\
\text { and } \\
\text { planning } \\
\text { industry } \\
\text { specific } \\
\text { programs. } \\
\text { Support } \\
\text { high } \\
\text { performin } \\
\text { g team } \\
\text { culture. } \\
\text { Provide } \\
\text { mentoring. }\end{array}$ \\
\hline $\begin{array}{l}2 \\
2\end{array}$ & $\begin{array}{l}\text { Eileen Fisher Inc. } \\
\text { (Entrepreneur \& } \\
\text { Fashion Designer) } \\
\text { NY, USA } \\
\text { www.eileenfisher.co } \\
\text { m } \\
\text { Employees: } 800+\end{array}$ & $\begin{array}{l}\text { Focuses } \\
\text { on the } \\
\text { provision } \\
\text { of high- } \\
\text { end } \\
\text { customer } \\
\text { service. } \\
\text { Rewards }\end{array}$ & $\begin{array}{l}\text { Reward } \\
\text { the faculty } \\
\text { for their } \\
\text { best } \\
\text { performan } \\
\text { ces. Share } \\
\text { the project } \\
\text { gains. }\end{array}$ \\
\hline
\end{tabular}

\begin{tabular}{|c|c|c|c|}
\hline & & $\begin{array}{l}\text { its } \\
\text { employee } \\
\text { s' } \\
\text { bonuses } \\
\text { for their } \\
\text { hard } \\
\text { work and } \\
\text { dedicatio } \\
\text { n up to } \\
\text { four } \\
\text { weeks of } \\
\text { earnings. } \\
\text { Remuner } \\
\text { ates for } \\
\text { personal } \\
\text { education } \\
\text { and } \\
\text { health } \\
\text { and } \\
\text { wellness } \\
\text { efforts. } \\
\text { Sets the } \\
\text { standard } \\
\text { for } \\
\text { creating } \\
\text { happy } \\
\text { employee } \\
\text { s, as a } \\
\text { result, } \\
\text { employee } \\
\text { loyalty is } \\
\text { at an all- } \\
\text { time } \\
\text { high. }\end{array}$ & $\begin{array}{l}\text { Reimburse } \\
\text { the cost of } \\
\text { undergoin } \\
\text { g faculty } \\
\text { developme } \\
\text { nt } \\
\text { programs. }\end{array}$ \\
\hline $\begin{array}{l}2 \\
1\end{array}$ & $\begin{array}{l}\text { Quicken Loans Inc. } \\
\text { (Mortgage, Lending) } \\
\text { Michigan, USA } \\
\text { www.quickenloans.c } \\
\text { om } \\
\text { Employees: } 17000\end{array}$ & $\begin{array}{l}\text { The } \\
\text { company } \\
\text { expresses } \\
\text { an active } \\
\text { appreciati } \\
\text { on for } \\
\text { creative } \\
\text { culture } \\
\text { and } \\
\text { encourag } \\
\text { es } \\
\text { employee } \\
\text { s to } \\
\text { innovate } \\
\text { without a } \\
\text { fear of } \\
\text { failure. } \\
\text { Even } \\
\text { trainees } \\
\text { are } \\
\text { empower } \\
\text { ed to } \\
\text { innovate } \\
\text { and } \\
\text { create on } \\
\text { a regular }\end{array}$ & $\begin{array}{l}\text { Eliminate } \\
\text { fear of } \\
\text { failure. } \\
\text { Encourage } \\
\text { creativity. } \\
\text { Guide to } \\
\text { undertake } \\
\text { sponsored } \\
\text { research. } \\
\text { Guide to } \\
\text { bid for } \\
\text { projects } \\
\text { under } \\
\text { MNCs and } \\
\text { IDAs. }\end{array}$ \\
\hline
\end{tabular}




\begin{tabular}{|c|c|c|c|}
\hline & & $\begin{array}{l}\text { basis. } \\
\text { The } \\
\text { company } \\
\text { believes } \\
\text { that when } \\
\text { people } \\
\text { are free } \\
\text { to create, } \\
\text { they do } \\
\text { remarkab } \\
\text { le things. } \\
\text { Combinin } \\
\text { g passion } \\
\text { with } \\
\text { creativity } \\
\text { causes } \\
\text { groundw } \\
\text { ork for } \\
\text { success. }\end{array}$ & \\
\hline 2 & $\begin{array}{l}\text { Hay MSL } \\
\text { (Real Estate), USA } \\
\text { www.hays.com }\end{array}$ & $\begin{array}{l}\text { MSL } \\
\text { pioneered } \\
\text { a } \\
\text { systemati } \\
\text { c way of } \\
\text { hiring the } \\
\text { best and } \\
\text { brightest. } \\
\text { It } \\
\text { implemen } \\
\text { ted panel- } \\
\text { based } \\
\text { interview } \\
\text { s and } \\
\text { psychom } \\
\text { etric } \\
\text { assessme } \\
\text { nt. }\end{array}$ & $\begin{array}{l}\text { Recruit } \\
\text { best and } \\
\text { the } \\
\text { brightest } \\
\text { faculty. } \\
\text { Do not } \\
\text { eliminate } \\
\text { their } \\
\text { achieveme } \\
\text { nts in the } \\
\text { comparati } \\
\text { ve } \\
\text { statements } \\
\text { for } \\
\text { recruitmen } \\
\text { t. }\end{array}$ \\
\hline $\begin{array}{l}1 \\
9\end{array}$ & $\begin{array}{l}\text { Mayo Clinic } \\
\text { (Medical Research } \\
\text { Group, Rochester, } \\
\text { Minnesota), USA } \\
\text { www.mayoclinic.org } \\
\text { Employees: } 63000\end{array}$ & $\begin{array}{l}\text { Reputed } \\
\text { for } \\
\text { employin } \\
\text { g the } \\
\text { brightest } \\
\text { minds to } \\
\text { offer } \\
\text { most } \\
\text { passionat } \\
\text { e service. } \\
\text { Promoted } \\
\text { team } \\
\text { dynamics } \\
\text { 60000 } \\
\text { employee } \\
\text { s link } \\
\text { arms with } \\
\text { one } \\
\text { another } \\
\text { to } \\
\text { provide } \\
\text { support } \\
\text { and care }\end{array}$ & $\begin{array}{l}\text { Encourage } \\
\text { supportive } \\
\text { culture. } \\
\text { Provide on } \\
\text { the job } \\
\text { training. }\end{array}$ \\
\hline
\end{tabular}

\begin{tabular}{|c|c|c|c|}
\hline & & $\begin{array}{l}\text { for } \\
\text { patients } \\
\text { and } \\
\text { families. } \\
\text { They } \\
\text { provide } \\
\text { extensive } \\
\text { on-the - } \\
\text { job } \\
\text { training. } \\
\text { The } \\
\text { employee } \\
\text { s receive } \\
\text { recogniti } \\
\text { ons for } \\
\text { their } \\
\text { efforts. }\end{array}$ & \\
\hline $\begin{array}{l}1 \\
8\end{array}$ & $\begin{array}{l}\text { Sainsbury's } \\
\text { (Online Shopping) } \\
\text { UK } \\
\text { www.sainsburys.co. } \\
\text { uk }\end{array}$ & $\begin{array}{l}\text { Company } \\
\text { takes the } \\
\text { lead in } \\
\text { HR } \\
\text { practices } \\
\text { for its } \\
\text { ability to } \\
\text { innovate } \\
\text { human } \\
\text { resource } \\
\text { practices. } \\
\text { Its people } \\
\text { strategy } \\
\text { is the } \\
\text { focus for } \\
\text { success. } \\
\text { Opened } \\
\text { its first } \\
\text { worker } \\
\text { academy } \\
\text { is the first } \\
\text { focus for } \\
\text { success. }\end{array}$ & $\begin{array}{l}\text { Develop } \\
\text { in-house } \\
\text { "Faculty } \\
\text { Academy" } \\
\text { and link it } \\
\text { with } \\
\text { Faculty } \\
\text { Developin } \\
\text { g } \\
\text { Institutes. }\end{array}$ \\
\hline $\begin{array}{l}1 \\
7\end{array}$ & $\begin{array}{l}\text { Hilcorp Energy Co. } \\
\text { (Exploration \& } \\
\text { Production) } \\
\text { Houston, USA } \\
\text { www.hilcorp.com } \\
\text { Employees: } 1381\end{array}$ & $\begin{array}{l}\text { Its focus } \\
\text { is a belief } \\
\text { in } \\
\text { mentorshi } \\
\text { p. Takes } \\
\text { focused } \\
\text { interest in } \\
\text { new } \\
\text { employee } \\
\text { s by } \\
\text { offering } \\
\text { mentorshi } \\
\text { p to } \\
\text { improve } \\
\text { the rate } \\
\text { of } \\
\text { success. } \\
\text { New } \\
\text { team } \\
\text { members }\end{array}$ & $\begin{array}{l}\text { Establish } \\
\text { "Mentorsh } \\
\text { ip } \\
\text { Programs" } \\
\text {. Pay } \\
\text { advance } \\
\text { increments } \\
\text { based on } \\
\text { the } \\
\text { excellence } \\
\text { like } \\
\text { earning } \\
\text { Ph.D. } \\
\text { degrees, } \\
\text { prior } \\
\text { teaching } \\
\text { experience } \\
\text { s and } \\
\text { Services }\end{array}$ \\
\hline
\end{tabular}




\begin{tabular}{|c|c|c|c|}
\hline & & $\begin{array}{l}\text { are } \\
\text { valued } \\
\text { and well- } \\
\text { trained } \\
\text { for } \\
\text { advance } \\
\text { ment. } \\
\text { Pays a } \\
\text { lucrative } \\
\text { wage. At } \\
\text { the end of } \\
\text { the } \\
\text { internship } \\
\text {, job } \\
\text { offers are } \\
\text { made. It } \\
\text { believes } \\
\text { in paying } \\
\text { it forward } \\
\text { when it } \\
\text { comes to } \\
\text { employee } \\
\text { charitable } \\
\text { contributi } \\
\text { ons. }\end{array}$ & $\begin{array}{l}\text { under } \\
\text { IDAs }\end{array}$ \\
\hline $\begin{array}{l}1 \\
6\end{array}$ & $\begin{array}{l}\text { Brigham and } \\
\text { Women's Hospital } \\
\text { (Boston, MA), USA } \\
\text { www.brighamandwo } \\
\text { men's.org }\end{array}$ & $\begin{array}{l}\text { Takes } \\
\text { efforts to } \\
\text { bring a } \\
\text { work and } \\
\text { life } \\
\text { balance } \\
\text { to the } \\
\text { communi } \\
\text { ty. It } \\
\text { encourag } \\
\text { es } \\
\text { employee } \\
\text { s to } \\
\text { balance } \\
\text { their } \\
\text { work and } \\
\text { personal } \\
\text { life. HR } \\
\text { focuses } \\
\text { on } \\
\text { childcare } \\
\text { for } \\
\text { working } \\
\text { mothers } \\
\text { and } \\
\text { fathers. } \\
\text { Subsidize } \\
\text { d tuition } \\
\text { is offered } \\
\text { for } \\
\text { employee } \\
\text { s who } \\
\text { earn } \\
\text { below a } \\
\text { certain }\end{array}$ & $\begin{array}{l}\text { Do not } \\
\text { stop } \\
\text { paying the } \\
\text { registratio } \\
\mathrm{n} \text { fees, \& } \\
\text { travel } \\
\text { grants for } \\
\text { presenting } \\
\text { papers in } \\
\text { the } \\
\text { internation } \\
\text { al } \\
\text { conference } \\
\text { s. Provide } \\
\text { nominatio } \\
\mathrm{n} \text { to take } \\
\text { up } \\
\text { internships } \\
\text { in foreign } \\
\text { universitie } \\
\text { s. }\end{array}$ \\
\hline
\end{tabular}

\begin{tabular}{|c|c|c|c|}
\hline & & $\begin{array}{l}\text { level. } \\
\text { Back-up } \\
\text { childcare } \\
\text { services } \\
\text { are also } \\
\text { offered in } \\
\text { the event } \\
\text { of } \\
\text { emergenc } \\
\text { ies. } \\
\text { Nursing } \\
\text { home } \\
\text { placemen } \\
\text { t, home } \\
\text { health } \\
\text { services, } \\
\text { and } \\
\text { support } \\
\text { groups } \\
\text { are part } \\
\text { of the } \\
\text { innovativ } \\
\text { e ways } \\
\text { the HR } \\
\text { team } \\
\text { which } \\
\text { helps its } \\
\text { employee } \\
\text { s } \\
\text { eliminate } \\
\text { stress. }\end{array}$ & \\
\hline $\begin{array}{l}1 \\
5\end{array}$ & $\begin{array}{l}\text { Diageo Plc. } \\
\text { (MNC Beverages } \\
\text { Co) UK } \\
\text { www.diadeo.com } \\
\text { Employees: } 29917\end{array}$ & $\begin{array}{l}\text { Highest } \\
\text { level of } \\
\text { integrity } \\
\text { and } \\
\text { innovatio } \\
\text { n even } \\
\text { during } \\
\text { times of } \\
\text { change. } \\
\text { Possess } \\
\text { long } \\
\text { heritage } \\
\text { of } \\
\text { systemati } \\
\text { c } \\
\text { leadershi } \\
\text { p } \\
\text { building. }\end{array}$ & $\begin{array}{l}\text { Do not } \\
\text { stop } \\
\text { nominatin } \\
\text { g for } \\
\text { global } \\
\text { training } \\
\text { programs } \\
\text { under } \\
\text { bilateral } \\
\text { schemes. }\end{array}$ \\
\hline $\begin{array}{l}1 \\
4\end{array}$ & $\begin{array}{l}\text { BT Group } \\
\text { (Telecommunication } \\
\text { s Holding Company, } \\
\text { MNC) London, UK } \\
\text { www.btplc.com } \\
\text { Employees: } 106400\end{array}$ & $\begin{array}{l}\text { During } \\
\text { credit } \\
\text { crunch } \\
\text { BT } \\
\text { retained, } \\
\text { retrained } \\
\text { and } \\
\text { redeploye } \\
\text { d existing } \\
\text { staff } \\
\text { instead of }\end{array}$ & $\begin{array}{l}\text { Provide } \\
\text { interdiscip } \\
\text { linary } \\
\text { training } \\
\text { programs } \\
\text { for the } \\
\text { faculty } \\
\text { members. } \\
\text { Provide } \\
\text { advanced } \\
\text { training in }\end{array}$ \\
\hline
\end{tabular}




\begin{tabular}{|c|c|c|c|}
\hline & & $\begin{array}{l}\text { laying } \\
\text { off. BT } \\
\text { allowed } \\
\text { their staff } \\
\text { to be } \\
\text { retained } \\
\text { in } \\
\text { different } \\
\text { areas, } \\
\text { gaining } \\
\text { valuable } \\
\text { experienc } \\
\text { e. } \\
\text { Apprentic } \\
\text { eship } \\
\text { programs } \\
\text { offering } \\
\text { flexible } \\
\text { work } \\
\text { schedules } \\
\text { are } \\
\text { available } \\
\text { to } \\
\text { employee } \\
\text { s who are } \\
\text { looking } \\
\text { for areas } \\
\text { of } \\
\text { movemen } \\
\text { t and } \\
\text { advance } \\
\text { ment } \\
\text { within } \\
\text { the } \\
\text { company. }\end{array}$ & $\begin{array}{l}\text { leadership } \\
\text { developme } \\
\text { nt and } \\
\text { student } \\
\text { personnel } \\
\text { developme } \\
\text { nt. }\end{array}$ \\
\hline $\begin{array}{l}1 \\
3\end{array}$ & $\begin{array}{l}\text { Cambridge } \\
\text { Consultants } \\
\text { (Product } \\
\text { Development, } \\
\text { Technology) } \\
\text { Cambridge, UK } \\
\text { www.cambridgecons } \\
\text { ultants.com } \\
\text { Employees: } 800\end{array}$ & $\begin{array}{l}\text { Company } \\
\text { provides } \\
\text { free } \\
\text { lunches. } \\
\text { It offers } \\
\text { unconven } \\
\text { tional } \\
\text { ways of } \\
\text { retaining } \\
\text { top talent. } \\
\text { It offers } \\
\text { staff high } \\
\text { degree of } \\
\text { autonomy } \\
\text { and } \\
\text { control } \\
\text { over their } \\
\text { career } \\
\text { trajectory } \\
\text {. Project - } \\
\text { led } \\
\text { structure } \\
\text { helps } \\
\text { employee } \\
\text { s gain }\end{array}$ & $\begin{array}{l}\text { Retain the } \\
\text { top talent } \\
\text { in } \\
\text { engineerin } \\
\mathrm{g} \\
\text { education. } \\
\text { Develop } \\
\text { multidisci } \\
\text { plinary } \\
\text { programs } \\
\text { for diverse } \\
\text { global } \\
\text { faculty } \\
\text { members. }\end{array}$ \\
\hline
\end{tabular}

\begin{tabular}{|c|c|c|c|}
\hline & & $\begin{array}{l}\text { training } \\
\text { and } \\
\text { experienc } \\
\text { e while } \\
\text { staying } \\
\text { relevant } \\
\text { to the } \\
\text { challenge } \\
\mathrm{s} \text { at task. } \\
\end{array}$ & \\
\hline $\begin{array}{l}1 \\
2\end{array}$ & $\begin{array}{l}\text { Marks \& Spencer } \\
\text { Plc. } \\
\text { (MNC-Retail) } \\
\text { London, UK } \\
\text { www.markandspenc } \\
\text { er.com } \\
\text { Employees: } 80787\end{array}$ & $\begin{array}{l}\text { Employe } \\
\text { e } \\
\text { engagem } \\
\text { ent puts } \\
\text { Marks } \\
\text { and } \\
\text { Spencer } \\
\text { at the top } \\
\text { of the } \\
\text { rung of } \\
\text { the } \\
\text { business } \\
\text { ladder. It } \\
\text { prioritize } \\
\text { d } \\
\text { communi } \\
\text { cation } \\
\text { builds the } \\
\text { backbone } \\
\text { of the } \\
\text { success } \\
\text { model. } \\
\text { The } \\
\text { company } \\
\text { has a } \\
\text { strong } \\
\text { reputatio } \\
\text { n in } \\
\text { employee } \\
\text { relations, } \\
\text { winning } \\
\text { awards } \\
\text { and } \\
\text { honors } \\
\text { for how } \\
\text { employee } \\
\text { s are } \\
\text { treated up } \\
\text { and down } \\
\text { the } \\
\text { supply } \\
\text { chain. At } \\
\text { Mars and } \\
\text { Spencer } \\
\text { there is } \\
\text { no such } \\
\text { thing as } \\
\text { too much } \\
\text { communi } \\
\text { cation. } \\
\text { From }\end{array}$ & $\begin{array}{l}\text { Develop e- } \\
\text { communic } \\
\text { ation, } \\
\text { technical } \\
\text { journals } \\
\text { and plan } \\
\text { round } \\
\text { table } \\
\text { meeting } \\
\text { on the } \\
\text { global } \\
\text { trends in } \\
\text { engineerin } \\
\text { g } \\
\text { education. } \\
\text { Plan } \\
\text { national } \\
\text { conference } \\
\text { s and } \\
\text { workshops } \\
\text { for } \\
\text { strategic } \\
\text { planning } \\
\text { and } \\
\text { institution } \\
\text { al } \\
\text { developme } \\
\text { nt. }\end{array}$ \\
\hline
\end{tabular}




\begin{tabular}{|c|c|c|c|}
\hline & & $\begin{array}{l}\text { regular } \\
\text { conferenc } \\
\text { e calls to } \\
\text { managem } \\
\text { ent to } \\
\text { daily } \\
\text { huddles } \\
\text { among } \\
\text { staff } \\
\text { Communi } \\
\text { cation is } \\
\text { key to the } \\
\text { success } \\
\text { of Marks } \\
\text { and } \\
\text { Spencer. }\end{array}$ & \\
\hline $\begin{array}{l}1 \\
1\end{array}$ & $\begin{array}{l}\text { USAA } \\
\text { (Insurance, Financial } \\
\text { Co), Texas, USA. } \\
\text { www.usaa.com } \\
\text { Employees: } 32896\end{array}$ & $\begin{array}{l}\text { The } \\
\text { company } \\
\text { is } \\
\text { recognize } \\
\text { d for its } \\
\text { retention } \\
\text { efforts } \\
\text { and } \\
\text { success. } \\
\text { It offers } \\
\text { annual } \\
\text { luncheon } \\
\text { s honor } \\
\text { ten } \\
\text { percent } \\
\text { of the } \\
\text { employee } \\
\text { s who } \\
\text { have } \\
\text { been with } \\
\text { the } \\
\text { company } \\
\text { over } 25 \\
\text { years. } \\
\text { The } \\
\text { retention } \\
\text { efforts } \\
\text { include } \\
\text { extensive } \\
\text { training } \\
\text { budgets, } \\
\text { bonuses, } \\
\text { and } \\
\text { intensive- } \\
\text { based pay } \\
\text { increase. } \\
\text { Less than } \\
4 \text { \% of IT } \\
\text { employee } \\
\text { s } \\
\text { turnover } \\
\text { and } 17 \% \\
\text { of the } \\
\text { employee }\end{array}$ & $\begin{array}{l}\text { Plan } \\
\text { outreach } \\
\text { global } \\
\text { programs } \\
\text { and } \\
\text { innovate } \\
\text { in MSME } \\
\text { consultanc } \\
\text { y } \\
\text { programs. } \\
\text { Plan } \\
\text { communit } \\
\text { y } \\
\text { developme } \\
\text { nt through } \\
\text { knowledge } \\
\text { capital and } \\
\text { plan } \\
\text { programs } \\
\text { under } \\
\text { various } \\
\text { internation } \\
\text { al } \\
\text { networkin } \\
\text { g. } \\
\text { Organize } \\
\text { MOOCs. } \\
\text { Plan } \\
\text { MMLPs } \\
\text { for } \\
\text { educationa } \\
1 \\
\text { leadership. }\end{array}$ \\
\hline
\end{tabular}

\begin{tabular}{|c|c|c|c|}
\hline & & $\begin{array}{l}\text { s } \\
\text { promoted } \\
\text { in } 2013 . \\
\text { It } \\
\text { provides } \\
\text { coffee } \\
\text { shops, } \\
\text { cafes, } \\
\text { relaxation } \\
\text { lounges, } \\
\text { fitness } \\
\text { centers } \\
\text { and } \\
\text { childcare } \\
\text { facilities. } \\
\text { Regular } \\
\text { social } \\
\text { events } \\
\text { create } \\
\text { camarade } \\
\text { rie } \\
\text { among } \\
\text { staff } \\
\text { members } \\
\text { and also } \\
\text { promote } \\
\text { the work- } \\
\text { life } \\
\text { balance } \\
\text { the } \\
\text { company } \\
\text { strives to } \\
\text { achieve. }\end{array}$ & \\
\hline $\begin{array}{l}1 \\
0\end{array}$ & $\begin{array}{l}\text { Rolls-Royce } \\
\text { (British Luxury } \\
\text { Automobile Maker, } \\
\text { Subsidiary of } \\
\text { BMW), UK. } \\
\text { www.rolls- } \\
\text { royce.com } \\
\text { Employees: } 50000\end{array}$ & $\begin{array}{l}\text { Long } \\
\text { synonym } \\
\text { ous with } \\
\text { quality } \\
\text { training } \\
\text { and } \\
\text { apprentic } \\
\text { eships. } \\
\text { Over } 30 \\
\text { \% of the } \\
\text { Rolls- } \\
\text { Royce's } \\
\text { senior } \\
\text { managers } \\
\text { started at } \\
\text { trainee- } \\
\text { level the } \\
\text { company. } \\
\text { The } \\
\text { business } \\
\text { model } \\
\text { and value } \\
\text { chain are } \\
\text { incredibl } \\
\text { y } \\
\text { innovativ } \\
\text { e. Rolls- }\end{array}$ & $\begin{array}{l}\text { Plan to } \\
\text { conduct } \\
\text { appreciativ } \\
\text { e appraisal } \\
\text { every year. } \\
\text { Plan tracer } \\
\text { studies } \\
\text { and impact } \\
\text { studies. } \\
\text { Plan } \\
\text { sponsored } \\
\text { studies on } \\
\text { the } \\
\text { performan } \\
\text { ce of } \\
\text { graduates. }\end{array}$ \\
\hline
\end{tabular}




\begin{tabular}{|c|c|c|c|}
\hline & & $\begin{array}{l}\text { Royce } \\
\text { showcase } \\
\text { s } \\
\text { transform } \\
\text { ation and } \\
\text { evolution } \\
\text {. Over } 80 \\
\% \text { of the } \\
\text { apprentic } \\
\text { es are } \\
\text { between } \\
16 \text { to } 18 \text {. } \\
\text { Most of } \\
\text { the } \\
\text { apprentic } \\
\text { es remain } \\
\text { and work } \\
\text { for the } \\
\text { company } \\
\text { to the life } \\
\text { of their } \\
\text { career. }\end{array}$ & \\
\hline 9 & $\begin{array}{l}\text { Shell } \\
\text { (British Dutch Shell } \\
\text { Plc.) } \\
\text { The Netherlands } \\
\text { www.shell.com } \\
\text { Employees: } 82000\end{array}$ & $\begin{array}{l}\text { Pioneered } \\
\text { internatio } \\
\text { nal } \\
\text { managem } \\
\text { ent } \\
\text { practices } \\
\text { by } \\
\text { involving } \\
\text { employee } \\
\text { s to } \\
\text { experienc } \\
\text { e in } \\
\text { working } \\
\text { in global } \\
\text { business. } \\
\text { It } \\
\text { believes } \\
\text { in having } \\
\text { a strong, } \\
\text { cohesive, } \\
\text { and open } \\
\text { culture } \\
\text { that } \\
\text { works } \\
\text { together } \\
\text { as a team } \\
\text { no matter } \\
\text { what } \\
\text { country } \\
\text { the team } \\
\text { member } \\
\text { is from. } \\
\text { Having a } \\
\text { global } \\
\text { mindset } \\
\text { is what } \\
\text { sets Shell } \\
\text { apart }\end{array}$ & $\begin{array}{l}\text { Plan } \\
\text { diverse } \\
\text { global } \\
\text { faculty } \\
\text { developme } \\
\text { nt center. } \\
\text { Plan } \\
\text { needed } \\
\text { short-term } \\
\text { and } \\
\text { medium- } \\
\text { term } \\
\text { programs } \\
\text { in human } \\
\text { resource } \\
\text { developme } \\
\text { nt and } \\
\text { manageme } \\
\text { nt. }\end{array}$ \\
\hline
\end{tabular}

\begin{tabular}{|c|c|c|c|}
\hline & & $\begin{array}{l}\text { from its } \\
\text { competito } \\
\text { rs. }\end{array}$ & \\
\hline 8 & $\begin{array}{l}\text { Ford Motor Co. } \\
\text { (American MNC) } \\
\text { Michigan, USA } \\
\text { www.ford.com } \\
\text { Employees: } 199000\end{array}$ & $\begin{array}{l}\text { HR } \\
\text { innovatio } \\
\mathrm{n} \text { through } \\
\text { a highly } \\
\text { discipline } \\
\mathrm{d} \text { culture, } \\
\text { outstandi } \\
\text { ng } \\
\text { training } \\
\text { opportuni } \\
\text { ties, and } \\
\text { strong } \\
\text { and } \\
\text { consistent } \\
\text { process. } \\
\text { It } \\
\text { believes } \\
\text { data and } \\
\text { discipline } \\
\text { should } \\
\text { come } \\
\text { first, } \\
\text { before } \\
\text { emotion. } \\
\text { When the } \\
\text { process is } \\
\text { adhered } \\
\text { to, a } \\
\text { healthy } \\
\text { work } \\
\text { environm } \\
\text { ent } \\
\text { succeeds. } \\
\text { People } \\
\text { managem } \\
\text { ent is } \\
\text { establishe } \\
\text { d in Ford. }\end{array}$ & $\begin{array}{l}\text { Develop } \\
\text { leadership } \\
\text { developme } \\
\text { nt } \\
\text { programs. } \\
\text { Offer } \\
\text { digital } \\
\text { technology } \\
\text {-based } \\
\text { programs. }\end{array}$ \\
\hline 7 & $\begin{array}{l}\text { Boston Consulting } \\
\text { Group } \\
\text { (Global Management } \\
\text { Consulting Group), } \\
\text { USA } \\
\text { www.bcg.com } \\
\text { Employees: } 18500\end{array}$ & $\begin{array}{l}\text { Average } \\
\text { hours of } \\
\text { training } \\
\text { per year } \\
\text { for full- } \\
\text { time } \\
\text { employee } \\
\text { s are } 100 . \\
\text { Hourly } \\
\text { employee } \\
\text { s receive } \\
\text { approxim } \\
\text { ately } 40 \\
\text { hours of } \\
\text { training } \\
\text { per year. } \\
\text { It is the } \\
\text { training } \\
\text { that }\end{array}$ & $\begin{array}{l}\text { Identify } \\
\text { in-house } \\
\text { faculty } \\
\text { developme } \\
\text { nt } \\
\text { programs. } \\
\text { Plan } \\
\text { mentorshi } \\
\text { p } \\
\text { programs } \\
\text { and } \\
\text { implement } \\
\text {. The } \\
\text { younger } \\
\text { faculties } \\
\text { are to be } \\
\text { trained in } \\
\text { consultanc } \\
\text { y works. }\end{array}$ \\
\hline
\end{tabular}




\begin{tabular}{|c|c|c|c|}
\hline & & $\begin{array}{l}\text { allows } \\
\text { employee } \\
\text { s of BCG } \\
\text { to } \\
\text { succeed. } \\
\text { Many } \\
\text { newcome } \\
\text { rs } \\
\text { graduate } \\
\text { to } \\
\text { leadershi } \\
\text { p } \\
\text { positions } \\
\text { within a } \\
\text { short } \\
\text { duration } \\
\text { of time. } \\
\text { mentorshi } \\
\text { ps and } \\
\text { open- } \\
\text { door } \\
\text { communi } \\
\text { cation } \\
\text { policies } \\
\text { with } \\
\text { leadershi } \\
\text { p allows } \\
\text { for } \\
\text { creative } \\
\text { freedom } \\
\text { and } \\
\text { confidenc } \\
\text { e to share } \\
\text { ideas. }\end{array}$ & \\
\hline 6 & $\begin{array}{l}\text { LinkedIN. } \\
\text { (American Business- } \\
\text { Employee } \\
\text { Oriented Co) } \\
\text { California, USA. } \\
\text { www.in.linkedin.co } \\
\text { m } \\
\text { Employees: } 15000\end{array}$ & $\begin{array}{l}\text { The } \\
\text { motto is } \\
\text { work } \\
\text { hard and } \\
\text { play hard. } \\
\text { The HR } \\
\text { team } \\
\text { hosts all- } \\
\text { company } \\
\text { parties, } \\
\text { complete } \\
\text { with live } \\
\text { music to } \\
\text { reward } \\
\text { staff } \\
\text { members } \\
\text { for their } \\
\text { hard } \\
\text { work and } \\
\text { dedicatio } \\
\text { n to the } \\
\text { company. } \\
\text { The team } \\
\text { dynamic } \\
\text { is } \\
\text { designed }\end{array}$ & $\begin{array}{l}\text { Invite new } \\
\text { proposals } \\
\text { for } \\
\text { industry } \\
\text { relevant } \\
\text { and } \\
\text { interdiscip } \\
\text { linary } \\
\text { programs. } \\
\text { Review } \\
\text { the } \\
\text { existing } \\
\text { programs } \\
\text { and invite } \\
\text { alumni to } \\
\text { know the } \\
\text { needed } \\
\text { programs } \\
\text { in } \\
\text { emerging } \\
\text { technology } \\
\text {. }\end{array}$ \\
\hline
\end{tabular}

\begin{tabular}{|c|c|c|c|}
\hline & & $\begin{array}{l}\text { to } \\
\text { encourag } \\
\text { e } \\
\text { employee } \\
\text { s to } \\
\text { create } \\
\text { systems } \\
\text { that break } \\
\text { the status } \\
\text { quo. } \\
\text { Once a } \\
\text { month, } \\
\text { the } \\
\text { company } \\
\text { holds an } \\
\text { InDay, } \\
\text { which is } \\
\text { when } \\
\text { employee } \\
\text { s set } \\
\text { aside } \\
\text { regular } \\
\text { new } \\
\text { ideas. } \\
\text { Employe } \\
\text { e } \\
\text { turnover } \\
\text { is less } \\
\text { than } 8 \% \\
\text { and } \\
\text { promotio } \\
\text { ns } \\
\text { consistent } \\
\text { ly over } \\
17 \% \text {. }\end{array}$ & \\
\hline 5 & $\begin{array}{l}\text { Twitter } \\
\text { (Social Networking } \\
\text { Service) } \\
\text { California, USA. } \\
\text { www.twitter.com } \\
\text { Employees: } 4600\end{array}$ & $\begin{array}{l}\text { It } \\
\text { promotes } \\
\text { work-life } \\
\text { balance, } \\
\text { causing } \\
\text { the } \\
\text { employee } \\
\text { s to } \\
\text { admire } \\
\text { the } \\
\text { company } \\
\text { culture. } \\
\text { HR } \\
\text { focuses a } \\
\text { lot of } \\
\text { time and } \\
\text { attention } \\
\text { to } \\
\text { training } \\
\text { managers } \\
\text { to lead, } \\
\text { while } \\
\text { maintaini } \\
\text { ng a } \\
\text { culture of }\end{array}$ & $\begin{array}{l}\text { Creating } \\
\text { work-life } \\
\text { balance; } \\
\text { The } \\
\text { institute } \\
\text { can } \\
\text { appoint a } \\
\text { manager } \\
\text { for faculty } \\
\text { training; } \\
\text { create a } \\
\text { culture of } \\
\text { authenticit } \\
\text { y, } \\
\text { transparen } \\
\text { cy, and } \\
\text { accessibilit } \\
\text { y. The } \\
\text { heads of } \\
\text { departmen } \\
\text { ts have to } \\
\text { be trained. }\end{array}$ \\
\hline
\end{tabular}




\begin{tabular}{|c|c|c|c|}
\hline & & $\begin{array}{l}\text { authentici } \\
\text { ty, } \\
\text { transpare } \\
\text { ncy, and } \\
\text { accessibil } \\
\text { ity. The } \\
\text { extensive } \\
\text { training } \\
\text { offered to } \\
\text { those in } \\
\text { leadershi } \\
\text { p. }\end{array}$ & \\
\hline 4 & $\begin{array}{l}\text { Nissan } \\
\text { (Nissan Co Ltd, } \\
\text { Japanese MNC), } \\
\text { Yokohama, Japan } \\
\text { www.nissan.in } \\
\text { Employees: } 138910\end{array}$ & $\begin{array}{l}\text { Blended } \\
\text { manufact } \\
\text { uring } \\
\text { technique } \\
\text { s of } \\
\text { Japanese } \\
\text { and } \\
\text { British } \\
\text { productiv } \\
\text { ity. The } \\
\text { key } \\
\text { philosoph } \\
\text { y is } \\
\text { Kaizen. It } \\
\text { empower } \\
\text { s the } \\
\text { workforc } \\
\text { e to } \\
\text { continuall } \\
\text { y } \\
\text { improve } \\
\text { the way } \\
\text { the job is } \\
\text { done. } \\
\text { Recruits } \\
\text { best } \\
\text { skilled } \\
\text { workers } \\
\text { and } \\
\text { support } \\
\text { them to } \\
\text { improve } \\
\text { their } \\
\text { skills. } \\
\text { The } \\
\text { company } \\
\text { has } \\
\text { implemen } \\
\text { ted } \\
\text { transpare } \\
\text { nt salary } \\
\text { scales } \\
\text { and } \\
\text { autonomy } \\
\text { for } \\
\text { leaders to } \\
\text { recruit } \\
\text { and build }\end{array}$ & $\begin{array}{l}\text { Similar to } \\
\text { Kaizen the } \\
\text { faculty } \\
\text { could be } \\
\text { trained in } \\
\text { managing } \\
\text { the faculty } \\
\text { for high } \\
\text { performan } \\
\text { ce; } \\
\text { Institutes } \\
\text { can recruit } \\
\text { the best } \\
\text { faculty } \\
\text { based on } \\
\text { their } \\
\text { accomplis } \\
\text { hments. }\end{array}$ \\
\hline
\end{tabular}

\begin{tabular}{|c|c|c|c|}
\hline & & $\begin{array}{l}\text { their own } \\
\text { teams. }\end{array}$ & \\
\hline 3 & $\begin{array}{l}\text { SAS } \\
\text { (Scandinavian } \\
\text { Airlines) } \\
\text { Stockholm, Sweden } \\
\text { www.sas.com }\end{array}$ & $\begin{array}{l}\text { It offers a } \\
\text { university } \\
\text { campus } \\
\text { feel on its } \\
\text { grounds, } \\
\text { with } \\
\text { greenway } \\
\text { s that } \\
\text { connect } \\
\text { buildings } \\
\text { to } \\
\text { buildings. } \\
\text { Recreatio } \\
\text { n and } \\
\text { fitness } \\
\text { facilities } \\
\text { are found } \\
\text { on } \\
\text { campus, } \\
\text { offering } \\
\text { time for } \\
\text { employee } \\
\text { s to take } \\
\text { part in } \\
\text { maintaini } \\
\text { ng the } \\
\text { health of } \\
\text { the entire } \\
\text { person- } \\
\text { mind, } \\
\text { body, and } \\
\text { spirt. It } \\
\text { offers a } \\
\text { high trust } \\
\text { environm } \\
\text { ent and } \\
\text { low } \\
\text { turnover } \\
\text { rate. HR } \\
\text { departme } \\
\text { nt is } \\
\text { addressin } \\
\text { g day-to- } \\
\text { day } \\
\text { stresses } \\
\text { and } \\
\text { concerns } \\
\text { that are } \\
\text { ent. } \\
\text { in a } \\
\text { eorkplac } \\
\text { enonm } \\
\text { anding }\end{array}$ & $\begin{array}{l}\text { Best } \\
\text { institution } \\
\text { al } \\
\text { planning } \\
\text { could be } \\
\text { undertaken } \\
\text {. } \\
\text { Recreation } \\
\text { al and } \\
\text { fitness } \\
\text { facilities } \\
\text { could be } \\
\text { established } \\
\text {. Faculties } \\
\text { are to be } \\
\text { guided to } \\
\text { maintain } \\
\text { healthy } \\
\text { mind and } \\
\text { spirt. }\end{array}$ \\
\hline 2 & $\begin{array}{l}\text { Cadbury } \\
\text { (British } \\
\text { Confectionery Co) } \\
\text { UK }\end{array}$ & $\begin{array}{l}\text { Puts its } \\
\text { people } \\
\text { first. It } \\
\text { maintaine }\end{array}$ & $\begin{array}{l}\text { Campus } \\
\text { could be } \\
\text { designed } \\
\text { to }\end{array}$ \\
\hline
\end{tabular}




\begin{tabular}{|c|c|c|c|}
\hline & $\begin{array}{l}\text { www.cadbury.co.uk } \\
\text { Employees: } 71657\end{array}$ & $\begin{array}{l}\mathrm{d} \text { worker } \\
\text { village } \\
\text { and R\&D } \\
\text { factories. } \\
\text { The } \\
\text { village } \\
\text { offers its } \\
\text { staff and } \\
\text { their } \\
\text { families a } \\
\text { comforta } \\
\text { ble } \\
\text { environm } \\
\text { ent to } \\
\text { work and } \\
\text { live. Its } \\
\text { culture } \\
\text { combines } \\
\text { positivity } \\
\text { and } \\
\text { balance, } \\
\text { among } \\
\text { work and } \\
\text { life. }\end{array}$ & $\begin{array}{l}\text { accommod } \\
\text { ate faculty } \\
\text { quarters, } \\
\text { playgroun } \\
\text { d for kids } \\
\text { and } \\
\text { school. }\end{array}$ \\
\hline 1 & $\begin{array}{l}\text { Google } \\
\text { (American MN Tech } \\
\text { Cop) } \\
\text { California, USA. }\end{array}$ & $\begin{array}{l}\text { Californi } \\
\text { a } \\
\text { headquart } \\
\text { ers offer }\end{array}$ & $\begin{array}{l}\text { There is a } \\
\text { need for } \\
\text { sports } \\
\text { facilities }\end{array}$ \\
\hline
\end{tabular}

\section{Discussion}

All the well performing companies intensively plan outstanding programs for improving the performance through training, evaluating the outcome and rewarding the workers based on the excellent accomplishment. The best employee development methods could be adopted with necessary changes to suit the higher education. It is presented in the following section.

\section{Synthesis}

\section{Institutional Development}

Institutional development centers around the faculty performance, motivation, delicateness, and support for their academic initiatives.

Table-3 Suggestions for New Initiatives

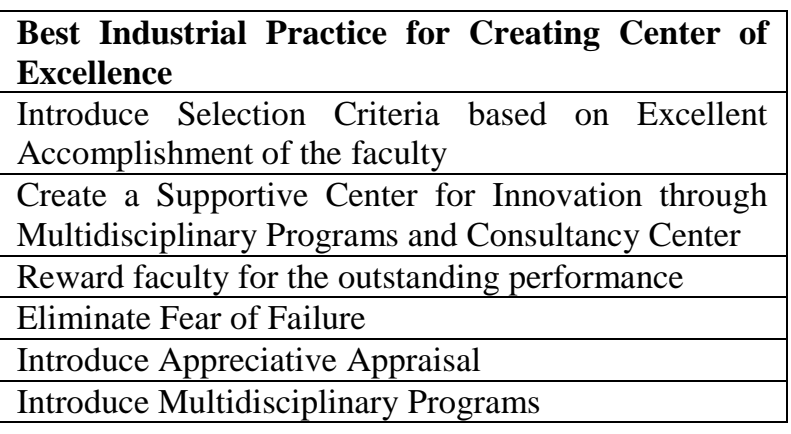

\begin{tabular}{|c|c|c|}
\hline $\begin{array}{l}\text { www.google.com } \\
\text { Employees: } 103459\end{array}$ & $\begin{array}{l}\text { a seven - } \\
\text { acre } \\
\text { sports } \\
\text { complex, } \\
\text { three } \\
\text { wellness } \\
\text { centers, } \\
\text { indoor } \\
\text { roller } \\
\text { hockey } \\
\text { rinks, } \\
\text { horseshoe } \\
\text { pits with } \\
\text { the right } \\
\text { tools, it } \\
\text { can } \\
\text { attract the } \\
\text { best } \\
\text { talent, } \\
\text { and } \\
\text { develop } \\
\text { happier } \\
\text { and more } \\
\text { productiv } \\
\text { e } \\
\text { employee }\end{array}$ & $\begin{array}{l}\text { and indoor } \\
\text { games. }\end{array}$ \\
\hline
\end{tabular}

\section{Faculty Development and Initiatives}

Investment in faculty development will provide outstanding returns through their excellence in planning industry specific programs, interdisciplinary graduate and postgraduate programs and undertaking sponsored projects etc.

Table-4 Suggestions for faculty Development Initiatives

\section{Faculty Development Practice}

Approve the faculty participation in global faculty development programs through IDAs/ Bilateral Schemes

Approve and reimburse the cost in participating in MOOCs organized by WBI, Coursera, edX, and other IDAs 


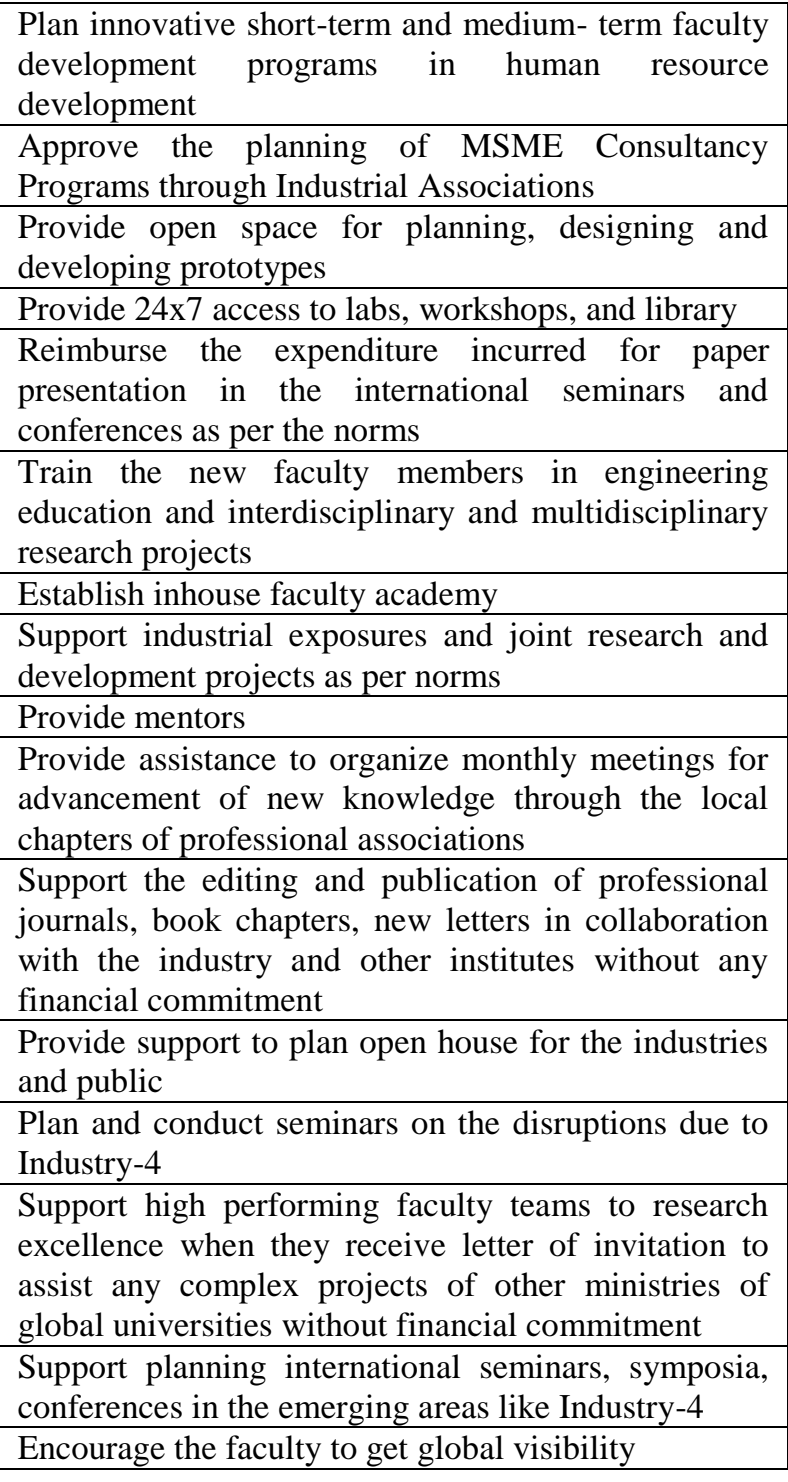

\section{Suggestions in Work Life Balance}

The faculty need to have very good health and they have to spend time with their kids and other family members. Based on the strength of faculty, the residential institutions provide needed resources.

Table-5 Suggestions in Work life Balance

\begin{tabular}{|l|}
\hline Taking Care of Work Life Balance \\
\hline Plan Staff Quarters with Necessary Services like \\
Water Supply, Street Light, Good Roads, Shopping \\
Center, Medical Facilities, \\
Elementary School, Middle School, High School, \\
Transport Facilities, Telephone Connections \\
\hline Playground \\
\hline Community Center \\
\hline Facilities for Indoor Games \\
\hline Park \\
\hline Fitness Center \\
\hline Leave Travel Concessions as per Rules \\
\hline Community hall for get together. \\
\hline
\end{tabular}

\section{Conclusion}

The best industries have achieved to get excellent returns on the bottom line through recognizing the employee needs. Most of the well performing higher education institutes which are governed by undisputed chairpersons, administrators, and leaders follow these human resource practices, but many poorly governed institutes do not implement various guidelines as per the rules and Acts. They have poor reputation and do not aid to create human and knowledge capital.

One remedial approach is to get periodical feedback from the faculty and discuss with the CEO. Suitable remedial measures should be taken to improve the academic ecosystem.

\section{References}

1. Agricultural College and Research Institute, Madurai, Tamil Nadu, India.

2. Bakthavatchalam Polytechnic College, Kanchipuram, Tamil Nadu, India

3. College of Engineering, Guindy, Chennai, India.

4. 2019 Creating a Work/Life Balance as Faculty Member, Retrieved from: https://www.research.fsu.edu/.../2019/2019creating-a worklife-balance-as-a facultymember

5. Faculty Work/Life Balance/Faculty, Retrieved from: https://hrs.boistate.edu/faculty/facultyworklife-balance

6. Faculty Work life Balance/Office of the Senior Vice... Retrieved from: https://www.provost.iastate.edu/.../isuadvance-resources/worklife-balance

7. Human Resources Management, MBA Staff (2015). The World's 30 Most Innovative Corporate Human, https://www.humanresourcesmba.net/worlds30-innovative-corporate-human-humanresource-development

8. Indian Institute of Technology, Madras, India, http://www.iitmc.ac.in

9. Retrieved fromhttps://www.humanresourcesmba.net/w orld-30-innovative-corporate-humanresources-departments/

10. Life Solutions/ Human Resources/ University of Pittsburgh, Retrieved from: www.hr.pitt.edu/.../work-lifebalance/lifesolutions 
11. University of Nebraska AdministrationWork-Life Balance, Retrieved from: www.nebrska.edu/faculty/work-life-balance

12. University of Pittsburgh, Retrieved from: www.provost.pitt.edu/sites/default/files/Facu lty

13. Work/life Balance/Bridgewater State University.

Retrieved from
http://www.bridgew.edu/.../worklife-balance

14. Work-life and Childcare/ It's Your Yale, Retrieved from http://your.yale.edu/your.yale.edu/.../benefits /work-life-and-childcare

15. Wellness \& Work-Life-hr.upenn.edu Retrieved from: www.hr.upenn.edu/PennHR/wellnessworklife

16. Work Life Balance/Center for Faculty Development-sjsu.edu Retrieved from: www.sjsu.edu/cfd/careerplanning/work-life-balance/index...

17. Mukhtar, Farah. (2012). Work life balance and job satisfaction among faculty at Iowa State University. Retrieved from: http://lib.dr.iastate.edu/etd/1279

18. Work Life Balance-University College Dublin, Retrieved from: www.ucd.ie/hr/benefitsforfacultyandstaff/wo rklifebalance-flexibleworking

19. Work-Life Balance/Office of the Provost/Penn Provost, Retrieved from: https://provost.upenn.edu/faculty/work-lifebalance

20. Wellness \& Work-Life-hr.upenn.edu Retrieved from: http://www.hr.upenn.edu/Penn HR/wellnessworklife/PennHR/wellness-work life 